\title{
Classification of Distal Radius Physeal Fractures Not Included in the Salter-Harris System
}

\author{
Nikolaos K. Sferopoulos * \\ Department of Pediatric Orthopaedics, Aristotle University of Thessaloniki, “G. Gennimatas” Hospital, 54635 \\ Thessaloniki, Greece
}

\begin{abstract}
Introduction: The most commonly used classification for pediatric physeal fractures has been proposed by Salter and Harris. Among the most suitable classification schemes are those proposed by Ogden and Peterson who added several new types of injuries. The purpose of this study was to examine the value of both schemes to classify all different types of physeal injuries of the distal radius that are not included in the Salter-Harris system and to test a new nomenclature to classify and guide treatment for the whole spectrum of these injuries.

Methods: A total of 292 children who were admitted for a physeal fracture of the distal radius that could not be classified according to the Salter-Harris system were identified from the hospital database. All radiographs were carefully examined and classified according to the existing classifications of Ogden and Peterson and a modified classification scheme. The results of the treatment were also evaluated.

Results: Ninety-six physeal injuries could not be classified using the classification schemes of Ogden and Peterson. All injuries could be classified in five types using the new, modified nomenclature. Growth abnormalities of the distal radius were evaluated after an average follow-up time of 11 years. Growth arrest due to a physeal bar was detected only in one patient.

Discussion: The proposed modified scheme is practical, incorporates all previous classification systems, allows classification of all physeal injuries of the distal radius that are not included in the Salter-Harris system and may assist comparison of treatment outcomes.
\end{abstract}

Keywords: Classification, distal radius, physeal fractures.

\section{INTRODUCTION}

The most popular classification for pediatric physeal fractures was proposed by Salter and Harris in 1963 [1]. It is based on the radiographic detection of the fracture line; it is practical and most suited for treatment planning. Ogden in 1981 [2] presented a new classification scheme partially based on the Salter-Harris system. He created more types and subtypes of injury patterns presenting a more extensive classification scheme that also included nonphyseal fractures as well as fractures complicated by localized damage to the growth plate leading to growth deformity. Peterson in 1994 [3] proposed a classification that included two new physeal fracture types and injuries of the Salter-Harris system under different enumeration. Ogden's more complex classification system as well as Peterson's description of new physeal fractures represents a considerable expansion of the SalterHarris classification. The use of both classification schemes has been proved to be of significant diagnostic and clinical importance [3, 4]. However, the simultaneous use of both Ogden and Peterson classifications has not been previously used in any clinical review. Moreover, it has not been previously reported whether the use of both classifications is

*Address correspondence to this author at the P. Papageorgiou 3, 546 35, Thessaloniki, Greece; Tel: 00302310 963270; Fax: 00302310 968265;

E-mail: sferopoulos@in.gr sufficient to include all physeal injuries that can not be included in the Salter-Harris classification system.

It has been shown that the lower end of the radius is the most common site of epiphyseal-plate injury if finger phalangeal physeal injuries are excluded $[1,5]$. Therefore, this study focused on whether the classification systems of Ogden and Peterson could include all physeal fractures of the distal radius that were not included in the Salter-Harris classification system. It also aimed to assess the value of the proposed new classification scheme to classify and to guide treatment for the entire wide spectrum of injuries.

\section{MATERIALS AND METHODS}

The radiographs of 1167 children that were admitted for acute fractures of the distal radius between 1984 and 2011 were, retrospectively, reviewed. Fractures were initially diagnosed as metaphyseal, physeal or fractures of the distal radius. Patients were admitted for bed rest and observation in the case of undisplaced fractures or for reduction of displaced fractures. Outpatient cases were not included in the study, since the radiographs of patients treated more than 2 years ago are usually recycled.

A metaphyseal fracture of the distal radius was diagnosed in 734 patients, while a physeal injury that could be classified according to the Salter-Harris system was diagnosed in 141 cases. 
This search identified 292 children with a physeal fracture that could not be classified according to the SalterHarris system. The average age in our 292 patients at injury was 10.8 years (range 3-14 years). There were 225 boys and 67 girls. Most patients (99\%) reported a fall-related injury that occurred at sport, school or home, while the rest were traffic-road accidents.

The radiographs of all cases were reviewed by the author twice with a 3-month interval. Diagnosis was not changed in any case during the second rating. The radiographic examination typically included anteroposterior and lateral radiographs of the distal forearm and wrist. In 11 cases, computed tomography was used to further delineate the fracture line. Magnetic resonance imaging was used only in one case with an epiphyseal extension of the fracture line.

Five types of physeal fractures, which could not be classified with the Salter-Harris system, were identified (Fig. 1). In type 1 injuries, a transverse fracture of the diaphysis or metaphysis, which involved the whole width of the cortex of the distal radius and was associated with a longitudinal linear fracture line extending to the physis, was evident. Displacement on type 1 injuries was seen only at the level of the transverse diaphyseal/metaphyseal fracture of the radius and was never evident within the distal fragment or at the level of the physis. In type 2 injuries, there was a transverse physeal fracture associated with a usually large metaphyseal fragment, which was subdivided by a fracture line that also extended to the physis. Displacement on type 2 injuries was seen at the level of the physeal fracture but was never evident within the metaphyseal fragment of the radius. In type 3 injuries, there was a physeal fracture associated with two metaphyseal fragments of the distal radial metaphysis one free that was always separated from the epiphysis and one attached to the epiphysis. Type 4 injuries were any of the type 1, 2 or 3 lesions associated with a fracture line extending beyond the physis to the epiphysis.

Finally, classification of any of the previous types complicated by asymmetric growth or premature physeal arrest was changed to a type 5 lesion.

\section{RESULTS}

After reviewing the radiological records of 1167 children admitted for acute fracture of the distal radius, a metaphyseal fracture was diagnosed in $734(63 \%)$ and a physeal injury that could be classified by the Salter-Harris system in 141 $(12 \%)$. An acute physeal injury of the distal radius that could not be classified by the widely accepted Salter-Harris system was diagnosed in 292 children (25\%).

The 292 patients were classified according to the classification proposed by Ogden and Peterson. An Ogden type 2B was diagnosed in 35 cases, while a Peterson type I lesion in 161 patients. Cases that could not be classified using the Ogden's classification were 257 and using the Peterson's 131, while 96 injuries could not fit to either of the two systems.

Using the newly proposed classification all physeal injuries that could not be included in the Salter-Harris classification scheme could be classified.

Type 1 lesion was evident in a total of 165 patients $(56.5 \%)$. The radiographic findings in only 4 patients indicated a transverse fracture line located to the distal diaphysis of the radius (Fig. $\mathbf{2 a}, \mathbf{b}$ ), while in the rest it was located to the metaphysis. A varying degree of angulation or displacement at the level of the transverse diaphyseal/ metaphyseal fracture was evident in 156 cases.

Type 2 lesion (Fig. 3) was evident in 88 patients $(30.13 \%)$. A varying degree of angulation or displacement at the level of the physeal fracture was evident in all patients.

All patients with type 1 or 2 lesions were treated conservatively.

Type 3 lesion (Fig. 4a, b) was evident in 35 patients $(12 \%)$. All lesions were displaced. A wide variation in the degree of displacement and the size of the metaphyseal fragments was evident in our patients. Metaphyseal fragments were neighboring or not. Additional fracture lines were evident in the free metaphyseal fragment in 3 patients. Imaging in them indicated the appearance of more than one free metaphyseal fragments. Diagnosis was based on radiographic findings. In 10 patients computed tomography was also used to secure diagnosis. Conservative treatment was applied in 28 patients, while 7 patients were treated with open reduction and internal fixation using Kirschner wires.

Type 4 lesion was evident in 3 patients (1.03\%). Intraepiphyseal extension of the fracture line in two of them

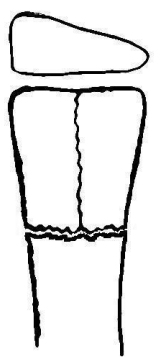

TYPE 1

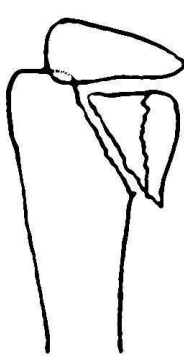

TYPE 2

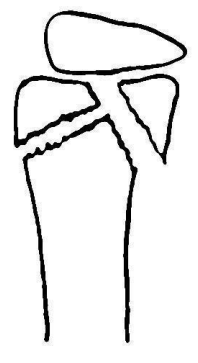

TYPE 3

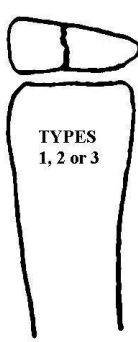

TYPE 4

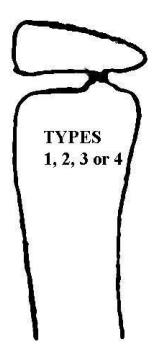

TYPE 5

Fig. (1). Schematic drawing of the classification system for physeal fractures of the distal radius not included in the Salter-Harris system. 
(a)

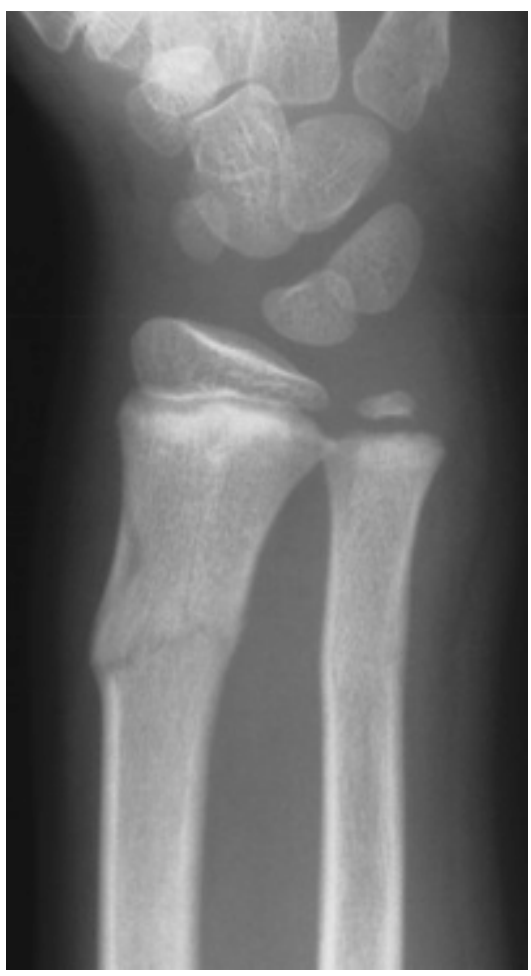

(b)

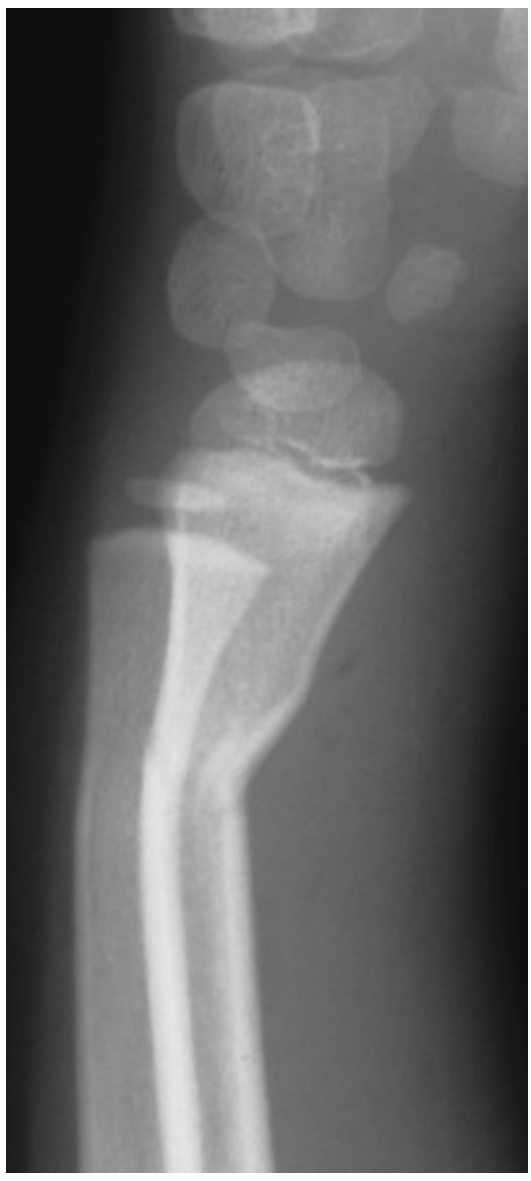

Fig. (2). Anteroposterior (a) and lateral (b) radiographs of a 10year-old boy indicated an angulated type 1 fracture.

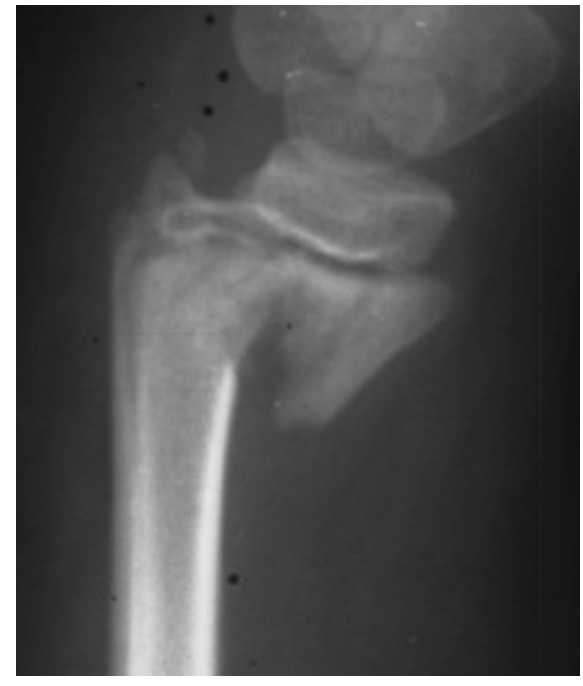

Fig. (3). Lateral radiograph of a 9-year-old girl indicated a displaced comminuted type 2 fracture.

(a)

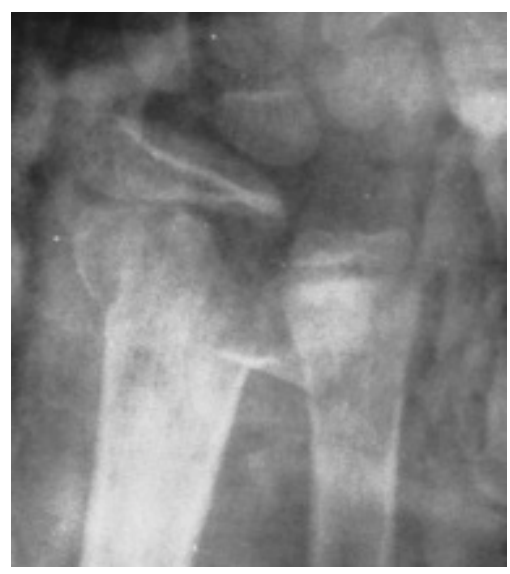

(b)

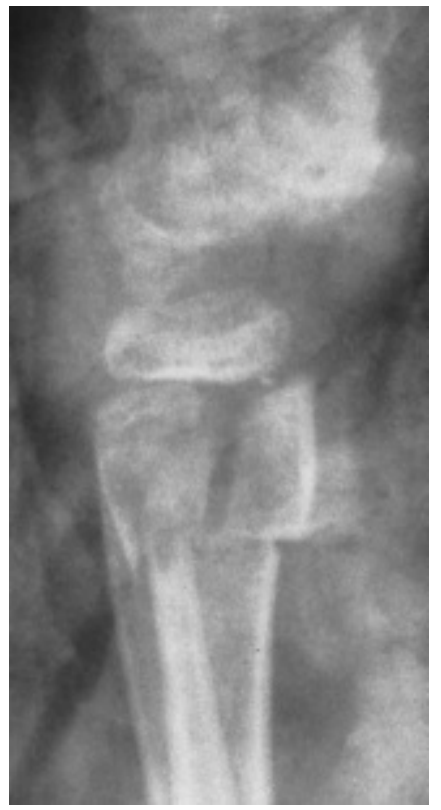

Fig. (4). Anteroposterior (a) and lateral (b) radiographs of an 11year-old boy indicated a displaced type 3 fracture. 
was associated with a type 1 (Fig. 5a) and in one case with a type 2 injury. All lesions were non-displaced. Diagnosis was based on radiographic findings. In 2 patients computed tomography and magnetic resonance imaging (Fig. 5b), respectively, were also used to secure diagnosis. All cases were treated conservatively.

(a)

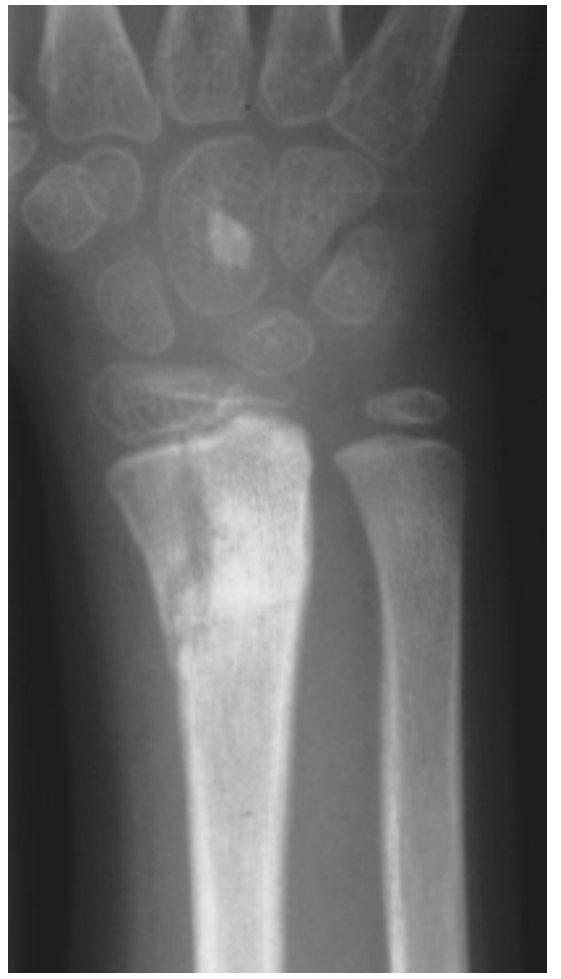

(a)

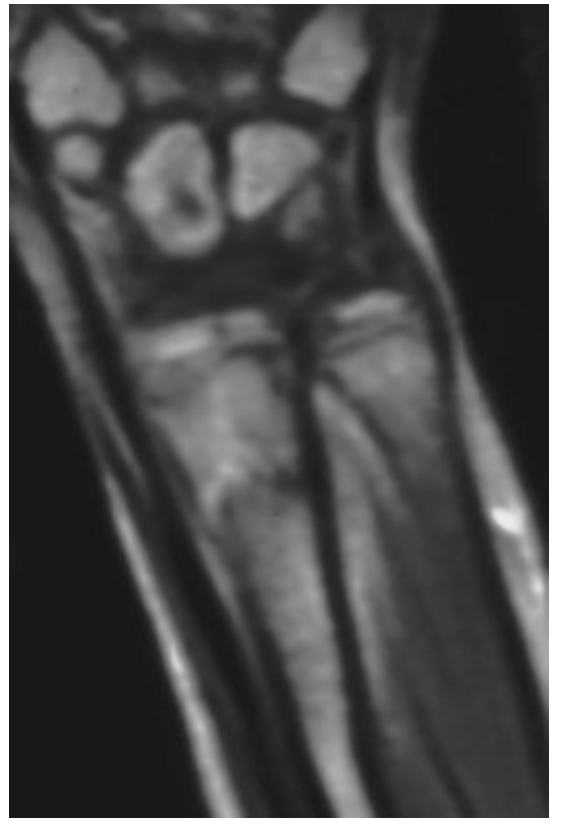

Fig. (5). Anteroposterior (a) radiograph of an 8-year-old girl indicated a transverse metaphyseal fracture with a longitudinal extension that crossed the physeal plate. Intraepiphyseal extension of the fracture line that was shown on $\mathrm{T} 1$ with contrast imaging (b) secured the diagnosis of an undisplaced type 4 lesion.
Both undisplaced and displaced fractures showed a degree of comminution. A type 1 or 2 lesion was considered to be comminuted, when there were two or more fracture lines within the distal fragment extending to the physis (Fig. 3). All type 3 lesions were considered to be a comminuted injury.

All injuries healed and showed complete remodeling of the distal radius on the serial radiographs taken during the post-injury period. After an average follow-up time of 11 years (range 2-26 years) no growth abnormalities of the distal radius were detected except for one patient with an initial type 1 (Fig. 6a, b) lesion that was complicated with radial growth arrest due to a physeal bridge (Fig. 6c). Classification in that patient was changed to a type 5 lesion.

\section{DISCUSSION}

In 1963, Salter and Harris classified epiphyseal injuries according to the direction of the fracture line as seen on plain radiographs [1]. Their classification gained immediate acceptance all over the world. Since then several additional physeal injuries have been described, which are distinctive and of potential clinical significance.

In 1981, Ogden proposed a more complex classification system consisting of nine fracture types and several subtypes [2]. Despite its great significance, it is not generally used in everyday practice $[3,6]$. However, its use was found recently to be significant to predict the incidence of premature physeal arrest following fractures of the distal forearm bones [4].

In 1994, Peterson with his classification added two new physeal fracture types. The first one, which was consistent with a transverse fracture through the metaphysis with a longitudinal extension towards the physis, but not along the physeal plate, was described as Peterson's type I lesion. This type of fracture showed a great variety of extensions of the fracture line from the metaphysis to the physis [7]. The second one, described as Peterson's type VI lesion, is in fact a Salter-Harris type IV injury in which a piece of the physis, usually accompanied with a portion of the epiphysis and metaphysis, is missing $[3,7]$.

Peterson's type I injury occurred most commonly in the distal radius with a frequency of $38.1 \%$ and was the second most common type of injury $(15.5 \%)$ only following SalterHarris type II injury [3, 7]. However, he concluded that the actual frequency might have been even greater, if radiographic examination of the metaphyseal fractures was more intensive, as well as if survey of the metaphyseal fractures had been included in his review.

In our study, from a total of 1167 cases, 292 patients were encountered with physeal fractures of the distal radius that could not follow the Salter-Harris classification. A total number of 96 patients could not be classified using either of the existing schemes of Ogden and Peterson. In the current study, a similar yet more inclusive classification scheme is proposed to label all various patterns of physeal injuries.

Type 1 injury is consistent with Peterson's description as type I injury, with the differentiation that the transverse fracture may also be located to the diaphysis. All the 
(a)

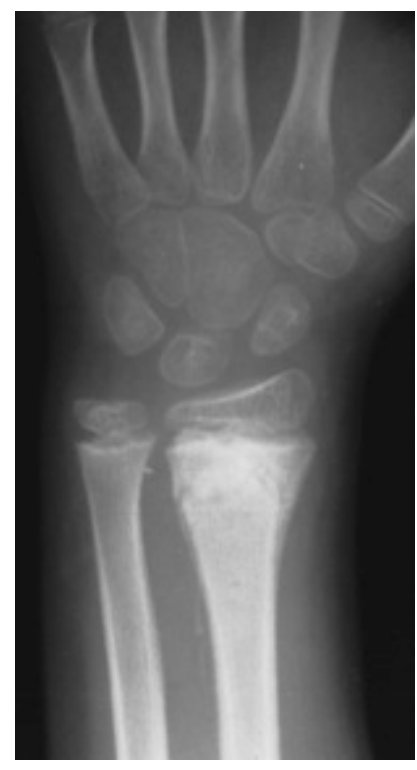

(b)

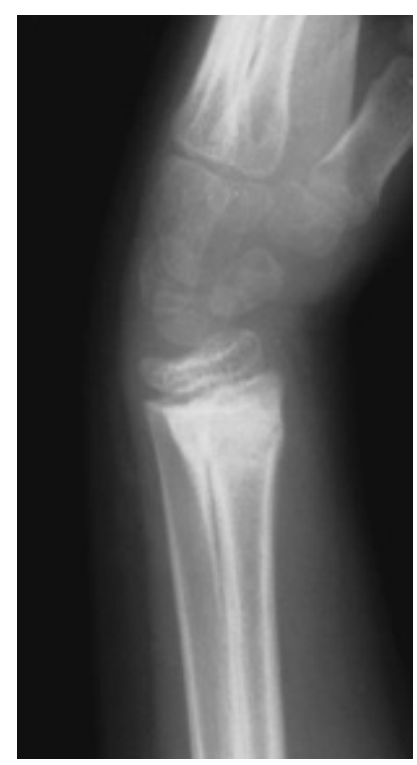

(c)

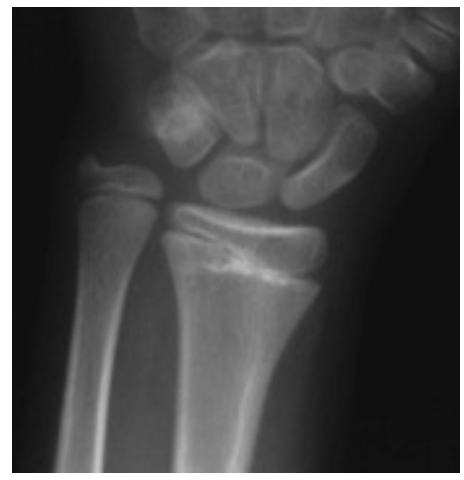

Fig. (6). Anteroposterior (a) and lateral (b) radiographs of a 9-yearold boy 5 weeks post-injury indicated a type 1 fracture with signs of periosteal healing. Diagnosis was changed to a type 5 lesion after the radiographic appearance of a bone bridge one year later $(\mathbf{c})$. inclusion criteria are according to Peterson [7], meaning that the fracture involves all cortical surfaces of the diaphysis or metaphysis and extends to the physis, but there is no fracture along the physeal plate, so attachment of the epiphysis with the metaphysis is not disrupted. The transverse diaphyseal or metaphyseal fracture may be angulated or displaced.

The radiographic findings in our patients were suggestive of a complete diaphyseal or metaphyseal transverse fracture on both frontal and lateral views. The linear longitudinal fracture line extending from the transverse fracture to the physis was usually evident on the anteroposterior view (Fig. 2a).

Type 2 injury is consistent with a Salter-Harris type II lesion, but its distinction lies on the radiological detection of an additional linear fracture line within the metaphyseal fragment, that remains attached to the epiphysis, extending to the physis. This metaphyseal linear fracture line, as with type 1 injury, does not separate or displace the fragments and it does not extend along the physeal plate. The physeal fracture may be angulated or displaced.

The radiographic findings in our patients were usually suggestive of a transverse metaphyseal fracture on the frontal view, whereas the triangular metaphyseal fragment that was usually seen on the lateral radiograph suggested a Salter-Harris type 2 fracture. This could be explained by the usually extensive borders of the triangular metaphyseal fragment. The linear fracture line, within the metaphyseal fragment, that extended to the physis was evident on either the anteroposterior or lateral view (Fig. 3).

Type 3 injury is consistent with Ogden's description as type 2B lesion. Propagation of the fracture forces, following a SalterHarris type II fracture, on the tensile side may lead to the creation of an additional free metaphyseal fragment, which is separated from the epiphysis. There may be a varying degree of displacement and the free metaphyseal fragment makes reduction much more difficult [8].

A wide variety in the size of the metaphyseal fragments and the degree of displacement was evident in this survey. The true size of the metaphyseal fragments was usually estimated after reduction. The fragments had either no metaphyseal contact at all or contact at the level of the physeal plate or metaphyseal borders. A varying degree of displacement was evident in our cases, since the metaphyseal fragment, which is separated from the epiphysis, may not be entirely separated from the metaphyseal bone. It may be reasonable to consider that additional fracture lines may be evident on both the attached and the free metaphyseal fragments, although in this report they were detected only in the free metaphyseal fragment. Radiographic diagnosis may occasionally be difficult. Computed tomography may be needed to secure diagnosis either when the size of the free metaphyseal fragment is very small or when it is big enough to differentiate from type 1 lesion indicating a portion of intact metaphyseal cortex.

Type 4 lesion is consistent with any of the previously described types associated with an extension of the fracture line along the epiphysis. The extension may not be clearly evident on conventional radiographs. Supplemental imaging, including computed tomography or magnetic resonance imaging may be needed to determine the precise fracture pattern. 
Furthermore, although it was not the subject of this review, it might be prudent to consider in the future whether other types of physeal injuries, not included in the Salter-Harris classification, such as the types 4B, C and D of the Ogden's classification and even the triplane fracture of the distal radius $[3,8,9]$ could also be included in the classification system presented in this study as type 4 lesions. Therefore, type 4 lesion could eventually include any type of physeal injury associated with a fracture line extending to the epiphysis that is not described in the Salter-Harris scheme.

In addition, any type of the previously described four types of physeal fractures complicated by a post-traumatic growth disturbance, due to asymmetric growth with or without the creation of a bony bar or bridge, is qualified as a type 5 injury. Growth disturbance following a physeal or nonphyseal injury has been adopted only by Ogden in his classification scheme $[2,8]$. The appearance of a bony bar may be erroneously considered as a late sequelae or complication of a physeal injury. However, it may be prudent to consider it as an event at the time of initial injury that exhibits a late radiographic appearance. It may be, therefore, reasonable to include it in a classification scheme of physeal injuries.

Physeal arrest following fractures of the distal radius is rare, with incidence ranging from $1 \%$ to $7 \%[10,11]$. The low incidence of growth arrest in this review may be related to the generalized application of the principles of treatment as described by Salter and Harris [1, 12, 13]. These were not followed in the single complicated case by means of repeated forceful attempts at closed reduction a couple of days following injury before his referral.

In the classification system reported in this review, type 1 injury was the most common one (56.5\%), and it was followed by type 2 lesion (30.13\%), type 3 lesion (12\%), type 4 lesion $(1.03 \%)$ and, finally, type 5 lesion $(0.34 \%)$.

There are several limitations in this report that should be considered when evaluating its value. In this study, the majority of cases were displaced. However, it is very likely that spectrum bias may exist, because undisplaced fractures are not usually referred and admitted or may even escape diagnosis. A candidate bias of the radiological evaluation may also occur since the whole estimation was performed by a single author.

This is the first attempt to describe a new reliable anatomic classification scheme of traumatic physeal injuries that could not be categorized by the Salter-Harris system. The most important drawbacks of the classification systems proposed by Ogden and Peterson are that they are not wide enough to include all types of injuries as well as the inclusion of fracture types already included in the Salter-Harris system. The latter makes description of a physeal injury more complicated, since it mandates the simultaneous clinical application of more than one classification systems.

The classification system presented in this report is supplemental to the Salter-Harris classification scheme concerning all not included types of fractures, where the distal radius has been taken into consideration. All included fracture types are precise and can be clearly differentiated from the lesions described in the Salter-Harris classification scheme. It incorporates all previous classification systems in only 5 types of physeal injuries. It is easily applied clinically and is, therefore, effective for rapid communication even for the nonexperienced trauma surgeon. It may also assist guiding and evaluating management and treatment outcomes. Finally, it correlates well with anatomic and epidemiologic fracture patterns and exhibits a logical progress from greater to lesser rate of occurrence. Epidemiological studies of physeal fractures with other localization than the distal radius will be required to show whether the reported classification system could be generally applicable to all physes of the immature skeleton.

\section{CONFLICT OF INTEREST}

The author certifies that he has no commercial associations (such as consultancies, stock ownership, equity interest, patent/licensing arrangements, etc) that might pose a conflict of interest in connection with the submitted article. The author received no financial support for this study.

\section{ACKNOWLEDGEMENTS}

Declared none.

\section{REFERENCES}

[1] Salter RB, Harris WR. Injuries involving the epiphyseal plate. J Bone Joint Surg Am 1963; 45(3): 587-622.

[2] Ogden JA. Injury to the growth mechanisms of the immature skeleton. Skeletal Radiol 1981; 6(4): 237-53.

[3] Peterson HA. Physeal fractures: Part 3. Classification. J Pediatr Orthop 1994; 14(4): 439-48.

[4] Cannata G, De Maio F, Mancini F, Ippolito E. Physeal fractures of the distal radius and ulna: long-term prognosis. J Orthop Trauma 2003; 17(3): 172-9.

[5] Peterson HA, Madhok R, Benson JT, Ilstrup DM, Melton LJ 3rd. Physeal fractures: Part 1. Epidemiology in Olmsted County, Minnesota, 1979-1988. J Pediatr Orthop 1994; 14(4): 423-30.

[6] Rogers LF, Poznanski AK. Imaging of epiphyseal injuries. Radiology 1994; 191(2): 297-308.

[7] Peterson HA. Physeal fractures: Part 2. Two previously unclassified types. J Pediatr Orthop 1994; 14(4): 431-8.

[8] Ogden JA. Skeletal growth mechanism injury patterns. J Pediatr Orthop 1982; 2(4): 371-7.

[9] Peterson HA. Triplane fracture of the distal radius: case report. J Pediatr Orthop 1996; 16(2): 192-4.

[10] Buterbaugh GA, Palmer AK. Fractures and dislocations of the distal radioulnar joint. Hand Clin 1988; 4(3): 361-75.

[10] Buterbaugh GA, Palmer AK. Fractures and dislocations of the distal radioulnar joint. Hand Clin 1988; 4(3): 361-75.

[11] Lee BS, Esterhai JL Jr, Das M. Fracture of the distal radial epiphysis. Characteristics and surgical treatment of premature, post-traumatic epiphyseal closure. Clin Orthop Relat Res 1984; 185: 90-6.

[12] Abzug JM, Little K, Kozin SH. Physeal Arrest of the Distal Radius. J Am Acad Orthop Surg 2014; 22(6): 381-9.

[13] Salter RB. Injuries of the epiphyseal plate. Instr Course Lect 1992; 41: 351-9. 